\title{
Las cargas superficiales y el flujo de energía en un circuito simple
}

(Surfaces charges and the energy's flow in a simple circuit)

\author{
Reinaldo Welti ${ }^{1}$ \\ Taller de Didáctica de la Ciencia y la Tecnología, Facultad de Ciencias Exactas e Ingeniería, Rosario, Argentina \\ Recebido em 15/10/2004; Revisado em 5/10/2005; Aceito em 17/10/2005
}

\begin{abstract}
El interés en el estudio de la distribución de las cargas superficiales sobre los conductores que transportan una corriente y su utilidad para la comprensión conceptual del comportamiento de los circuitos se han incrementado recientemente. Numerosos trabajos y textos presentan resultados cualitativos, analíticos y numéricos para diferentes geometrías de la estructura del circuito. Se presentan aquí los resultados analíticos para un circuito simple formado por placas rectangulares. Esta geometría permite determinar no solo la distribución de las cargas superficiales que crean el campo en el interior de los conductores, sino también el flujo de energía que se dirige desde la fuente hasta los elementos resistivos del circuito.

Palabras clave: circuitos, cargas superficiales, campo eléctrico, flujo de energía.
\end{abstract}

The interest in the study of the superficial charges's distribution on the conductors that transport a current and its utility for the conceptual comprehension of the circuits's behavior have been increased recently. Numerous works and texts present numerical, analytic, and qualitative results for different geometries of the circuit's structure. The analytic results for a simple circuit formed by rectangular plates are presented here. This geometry permits to determine not only the distribution of the superficial charges that create the field in the interior of the conductors, but also the flow of energy that is directed since the source to the resistive elements of the circuit.

Keywords: circuits, surfaces charges, electric field, flow of energy.

\section{Introducción}

En un curso tradicional de electricidad, la teoría de circuitos de corriente continua se formula en términos de los conceptos de potencial, carga y corriente, mientras que los campos electromagnéticos juegan un rol secundario o nulo. Esto hace que los campos y los circuitos aparezcan como dos tópicos completamente diferentes y no relacionados. Sin embargo, un circuito simple, como el formado por una batería y una resistencia, tienen una física realmente interesante que la mayoría de los textos omiten.

Una posibilidad de unificar el tratamiento de los campos y circuitos es subrayar el rol desempeñado por las cargas en la superficie de los elementos conductores del circuito en la creación del campo eléctrico en el interior de estos elementos. Como lo dice Jackson [1]: "La importancia de las cargas superficiales asociadas a las corrientes en un circuito no es generalmente reconocida. En general, los conductores de un circuito con corriente deben tener una distribución de carga superficial no uniforme." Es un problema de la teoría de campos explicar cómo esta distribución de cargas super-

\footnotetext{
${ }^{1}$ E-mail: weltreb@amet.com.ar.

Copyright by the Sociedade Brasileira de Física. Printed in Brazil.

ficiales crea un campo eléctrico uniforme en el interior del hilo. La mayoría de los libros de física pasan por alto esta cuestión. La existencia de una distribución de cargas superficiales sobre la superficie de los conductores está sugerida en Scott [2]; Sommerfeld [3] hace un cálculo detallado para un cable coaxil cuyo conductor interno es resistivo y el externo conductor perfecto, mientras que Reitz y Milford [4] parecen sugerir que ninguna carga superficial puede existir en un circuito de corriente continua.

La importancia de las cargas superficiales en la formulación de la teoría de circuitos ha sido revitalizada por Chabay y Sherwood [5] en un libro de física destinado a estudiantes del ciclo básico de ciencias e ingeniería. Los autores analizan la relación entre la distribución de cargas superficiales y los campos eléctricos basados en un trabajo de Haertel [6] que describe la descarga de un capacitor a través de un circuito altamente resistivo. Más recientemente, Prayer [7] presenta cálculos numéricos de las cargas superficiales para los diferentes circuitos que [5] y [6] utilizan en sus ejemplos cualitativos.

Se han obtenido soluciones analíticas para algunas 
geometrías relativamente simples: Sommerfeld [1] considera un cable coaxial infinito con un conductor interno resistivo y el externo de conductividad infinita. Russell [8] generaliza los resultados de Sommerfeld para un sistema de conductores paralelos e infinitos, mientras que Ferreira y Figueredo [9] adaptan el análisis de Sommerfeld para un cable coaxial semi-infinito cerrado en uno de sus extremos por un disco perfectamente conductor. Jefimenko [10] calcula los campos en el interior de un circuito formado por placas paralelas, Assis et al. [11] deducen los campos afuera de las placas y Assis et al. [12], analizan las cargas superficiales y los campos de un hilo resistivo rectilíneo de sección circular. Heald [13] encuentra los campos afuera y adentro de un circuito cilíndrico con corriente azimutal y el flujo de energía que va desde la batería hacia la superficie del cilindro resistivo. Hernández et al. [14] calculan las cargas superficiales y el campo eléctrico de una superficie toroidal resistiva con corriente azimutal.

El objetivo de este trabajo es utilizar los numerosos trabajos numéricos y analíticos que hemos mencionado más arriba para presentar una propuesta, dirigida especialmente a los profesores universitarios de física, sobre cómo tratar estos temas en el aula. En la sección 2 hacemos una descripción del establecimiento de una corriente estacionaria en un conductor, en la 3 se resumen los resultados obtenidos en [10], que se utilizan, luego, en la sección 4 para dar una explicación cualitativa de la relación entre la distribución de cargas superficiales y los campos eléctricos en el interior y exterior de los hilos conductores de un circuito. Finalmente, en la sección 5, se consideran las cargas superficiales y el flujo de energía en un circuito simple compuesto de una batería y una resistencia.

\section{La formación de una corriente esta- cionaria en un conductor}

El movimiento de cargas en el interior de un conductor se describe mediante el campo vectorial $\boldsymbol{J}$, denominado densidad de corriente o flujo de cargas (la carga que atraviesa la unidad de área en la unidad de tiempo). El vector densidad de corriente en un punto de un conductor homogéneo e isotrópico es proporcional al campo eléctrico $\boldsymbol{E}$, es decir $\boldsymbol{J}=\boldsymbol{E} / \rho$, donde $\rho$, la resistividad, es una constante escalar característica del material.

Si el flujo es estacionario tanto $\boldsymbol{J}$ como $\boldsymbol{E}$ deben ser paralelos a la superficie de conductor. En efecto, si $\boldsymbol{J}$ tiene una componente no nula en un punto de la superficie del conductor, se estaría acumulando carga en ese punto lo que produciría una variación del campo eléctrico y, por lo tanto, de la densidad de corriente, lo cual contradice la hipótesis de flujo estacionario. Las corrientes estacionarias requieren además que el circuito sea cerrado. Debido a las pérdidas de energía en el conductor, el mantenimiento de una corriente estacionaria requiere una fuente de energía externa, como por ejem- plo una batería química. Surge entonces la siguiente pregunta: ¿cómo viaja la energía desde la batería donde está disponible hasta las porciones del circuito donde ésta se disipa en forma de calor? Esta cuestión será abordada en la seccion 5 .

Se analiza a continuación cómo se llega a establecer una corriente estacionaria en un circuito eléctrico considerando las etapas que se muestran esquemáticamente en la Fig. 1. Inicialmente no hay hilos conductores conectados a los dos electrodos de la batería (Fig. 1a). Se conectan luego dos hilos rígidos largos a los dos terminales (Fig. 1b). Después que las cargas a lo largo de estos conductores llegan al estado estacionario se unen los extremos de estos hilos (Fig. 1c) y la corriente comienza.

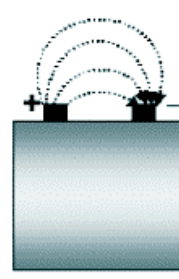

(a)

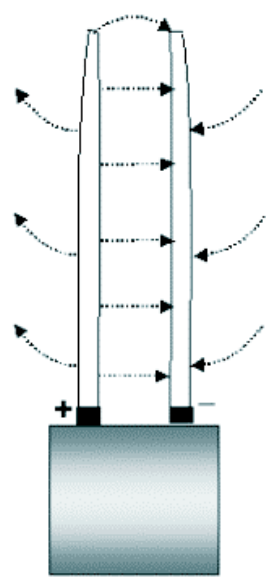

(b)

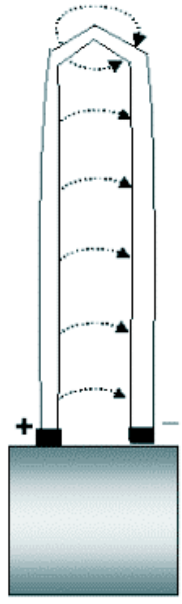

(c)
Figura 1 - Líneas de fuerza del campo eléctrico alrededor de una batería (a) y alrededor de los hilos conectados a la misma antes (b) y después de unirlos en sus extremos superiores.

En la primera etapa, como se muestra en la Fig. 1a, se tiene un campo eléctrico "dipolar" entre los terminales de la batería (se muestra solamente el campo eléctrico en la zona externa de la batería). En la segunda etapa, Fig. 1b, los dos hilos resultan extensiones de los volúmenes equipotenciales de cada uno de los electrodos. No hay nada que impida que estos hilos tengan una forma arbitraria o una extensión de varios kilómetros o que se doblen o que se retuerzan uno alrededor del otro ( $\sin$ tocarse). Las líneas de fuerza del campo eléctrico nacen en la superficie de uno de los hilos y terminan en el otro, en ángulo recto.

Cuando se acercan los extremos de los hilos, el campo eléctrico en estos extremos incrementa a un gran valor (igual diferencia de potencial, distancia más pequeña), dando lugar en muchos casos a la producción de una chispa justo antes de que se toquen. En el instante en que se ponen en contacto se produce un movimiento de electrones bajo la acción de este campo grande, destruyendo la naturaleza equipotencial de los interiores de los dos conductores. De esta manera, se 
produce el inicio de la corriente. Parte del flujo de corriente tiene en esta etapa componentes perpendiculares a los contornos del conductor que van modificando la distribución de cargas superficiales que son, a su vez, las responsables de encauzar la corriente a lo largo de los hilos (esto es, hacerlos paralelos a la superficie). Estos efectos comienzan en los extremos que se han puesto en contacto y se extienden a lo largo de toda la longitud del hilo hasta llegar a los terminales de la batería. El proceso de reordenamiento se dirige ahora en la dirección inversa, desde los terminales de la batería hasta los extremos que han sido puestos en contacto. Este proceso de reajuste en ambas direcciones continúa hasta que se llega al estado estacionario. El tiempo que se tarda en llegar al estado estacionario es experimentalmente muy corto.

En esta etapa, como se muestra en la Fig. 1c las líneas de fuerza del campo eléctrico salen y entran en la superficies de los conductores en ángulos diferentes de $90^{\circ}$ debido a que se tiene ahora una componente de campo eléctrico paralelo a los hilos. Esta componente del campo eléctrico "penetra" en el interior del hilo y da origen a la corriente que circula a lo largo del mismo.

En la próxima sección vamos a determinar los campos eléctricos y las cargas superficiales que rodean al circuito en el caso especial en que los "hilos" conductores son dos placas de longitud y ancho grandes comparadas con su separación.

\section{La carga superficial y el campo eléctrico en un circuito de placas paralelas}

En lugar de los dos hilos de la Fig. 1, se conectan a los bornes de la batería dos placas conductoras paralelas, de resistividad $\rho$, separadas por una distancia $d \mathrm{y}$ de dimensiones: ancho $a$, longitud $l$ y espesor $e$. Si $l$ y $a$ son mucho mayores que $d$, podemos despreciar los "efectos de borde", esto es, se puede suponer que las cargas superficiales se distribuyen solamente en sus superficies internas. La solución analítica completa de este problema se puede encontrar en la referencia [10]. En esta sección vamos a comentar los resultados que se relacionan con los pasos 2 y 3 de la sección anterior.

Después de conectar las placas a los bornes de la batería, éstas se cargan eléctricamente, positivamente, la que está conectada al borne positivo y negativamente, la otra. El interior de cada una de las placas es un volumen equipotencial y la diferencia de potencial entre ambas, $V_{0}$, coincide con la fem de la batería ideal (sin resistencia interna). Si despreciamos los efectos de borde, el campo eléctrico entre las placas viene dado por

$$
E_{y}=\frac{V_{0}}{d}
$$

y las cargas superficiales en las superficies $y=0$ e $y=d$, que vienen dadas por

$$
\begin{aligned}
& \sigma(y=0)=\varepsilon_{0} \frac{V_{0}}{d} \\
& \sigma(y=b)=-\varepsilon_{0} \frac{V_{0}}{d}
\end{aligned}
$$

Se observa que la densidad de carga no depende de la resistividad del material.

Se unen ahora las dos placas, en $z=0$, mediante una placa perfectamente conductora como se muestra en la Fig. 2 (una sección del circuito perpendicular al eje $x$ ). Después de producido el contacto, se establece un régimen transitorio similar al que se describió en la etapa 3 de la sección anterior. Una vez que se llega al estado estacionario, circula, a través del circuito, una corriente constante $I=V_{0} / R$, donde $R=\rho \mathscr{L l} / S$ es la resistencia total del circuito y $S=a e$ es la sección de cada una de las placas. El módulo del campo eléctrico en el interior de las placas resistivas, de acuerdo a la ley de Ohm, es $\rho I / S$ y su dirección y sentido coincide con la de la corriente que circula a lo largo de las mismas.

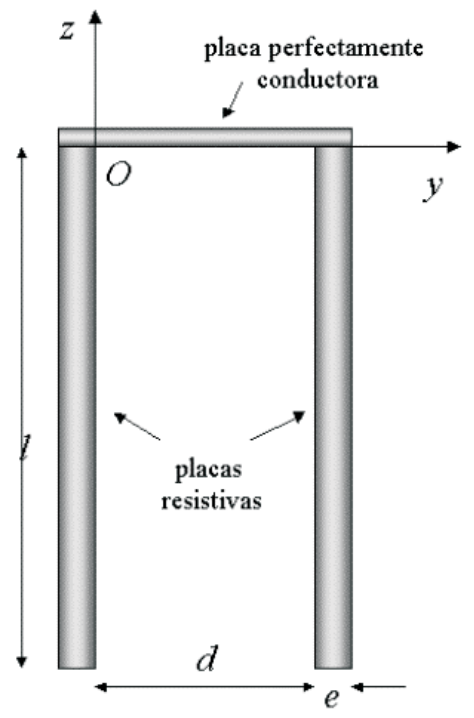

Figura 2 - Dos placas resistivas paralelas, unidas en $z=0$ por una placa perfectamente resistiva.

El potencial en el interior de las placas que satisface la ecuación de Laplace y las condiciones de contorno: $\partial \Phi / \partial z=-\rho I / S$ y $\partial \Phi / \partial z=-\rho I / S$, sobre las superficies de las placas resistivas, $y=0$ e $y=d$, respectivamente y $\partial \Phi / \partial y=0$, sobre la superficie de la placa perfectamente conductora, viene dado por

$$
\Phi(y, z)=\frac{\rho I}{S}\left(\frac{2 y}{d}-1\right) z
$$

si se supone que la placa perfectamente conductora en $z=0$, está conectada a tierra.

El campo eléctrico entre las placas $(0<y<d)$ es, 


$$
\begin{gathered}
E_{y}=-\frac{\partial \Phi}{\partial y}=-\frac{2 \rho I z}{S d} \\
E_{z}=-\frac{\partial \Phi}{\partial z}=-\frac{2 \rho I y}{S d}+\frac{\rho I}{S}
\end{gathered}
$$

El salto de la componente normal a las placas, en $y=0$ e $y=d$, indica la existencia de cargas superficiales sobre estas superficies:

$$
\begin{gathered}
\sigma(y=0)=\varepsilon_{0} E_{y}(y=0)=-\varepsilon_{0} \frac{2 \rho I z}{S d} \\
\sigma(y=d)=-\varepsilon_{0} E_{y}(y=d)=\varepsilon_{0} \frac{2 \rho I z}{S d}
\end{gathered}
$$

Se observa que el módulo de la densidad de carga superficial decrece linealmente con $z$, es máxima en $z=-l$ $\mathrm{y}$ es cero en $z=0$. Este gradiente de la densidad de carga superficial es la causante de la componente tangencial del campo eléctrico en la superficie de la placa resistiva y del campo en el interior de la placa que origina la corriente que circula a lo largo de la misma.

En la Fig. 3 se muestran las formas de las líneas de fuerza del campo eléctrico en el espacio entre las dos placas: las líneas de campo son normales a la placa perfectamente conductora, pero no lo son sobre las dos placas resistivas.

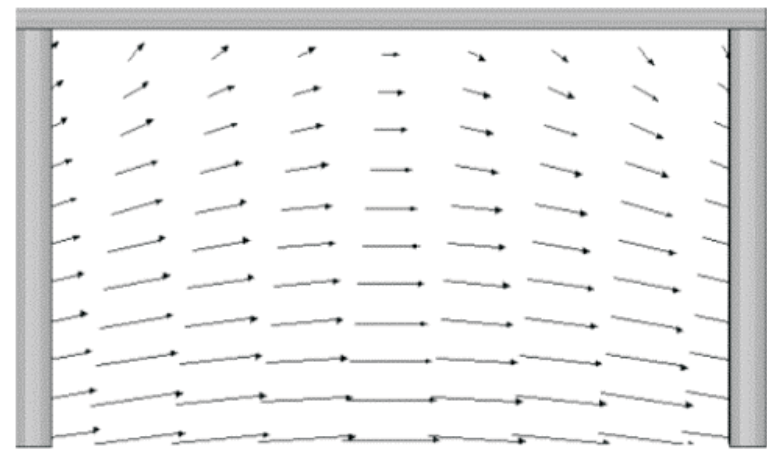

Figura 3 - Campo eléctrico en el interior del circuito.

Si expresamos las Ecs. 13 en términos de la diferencia de potencial $V_{0}=R I=(2 \rho l / S) I$ obtenemos:

$$
\sigma(y=0, z)=-\varepsilon_{0} \frac{V_{0}}{d} \frac{z}{l} \quad \sigma(y=d, z)=\varepsilon_{0} \frac{V_{0}}{d} \frac{z}{l}
$$

Se observa que la densidad de carga superficial varía linealmente desde su valor máximo (en módulo) en $z=-l$, igual al valor que tenía antes de cortocircuitar las placas, hasta cero en el cortocircuito $z=0$. Esto significa que en el proceso de distribución de cargas que se produce después de cortocircuitar las placas, la carga total de cada una de ellas disminuye a la mitad.

El campo magnético en el interior de las placas es el de un solenoide infinito: su módulo es $I / a$ y su dirección es perpendicular al papel (entrante). Afuera de las placas el campo magnético es cero y en el interior de las chapas varía linealmente desde su valor $I / a$ en la superficie interna hasta cero en la superficie externa.

El vector de Poynting $\boldsymbol{P}=\boldsymbol{E} \times \boldsymbol{H}$, en cada punto del espacio, es ortogonal a las líneas de fuerza del campo eléctrico $\boldsymbol{E}$. Las líneas del flujo de energía coinciden, por lo tanto, con las líneas equipotenciales (las líneas continuas que se muestran en la Fig. 4). El sentido de las líneas de flujo van desde la batería a los elementos resistivos del circuito. El vector de Poynting tiene una componente normal no nula (y hacia adentro) solamente en las superficies de las placas resistivas.

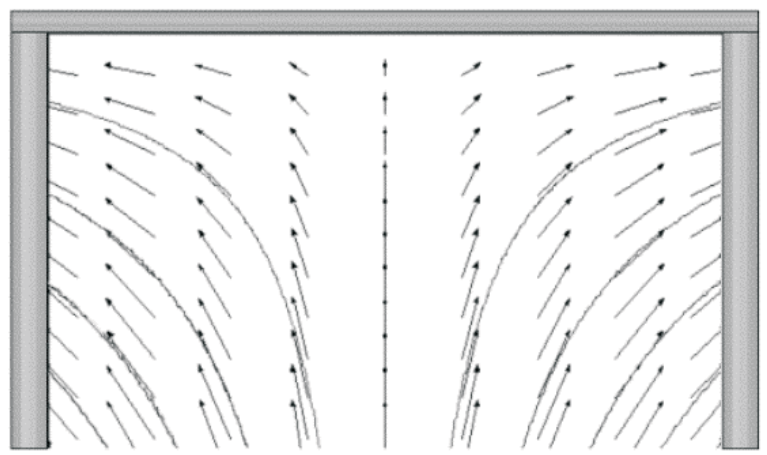

Figura 4 - Vector de Poynting y líneas equipotenciales.

Como las dimensiones de las placas son infinitas en la dirección $x$, el campo magnético es nulo en el exterior del circuito, sin embargo, como las componentes tangenciales del campo eléctrico sobre la superficie externa de las placas resistivas no es nula existe un campo eléctrico externo. El campo eléctrico externo se calcula en la referencia [13] para un circuito cilíndrico de sección circular y en la referencia [10] para un par de placas paralelas similar al de la Fig. 2.

$\mathrm{Si}$ las dimensiones de las placas no son infinitas a lo largo el eje $x$, existe un campo magnético en el exterior de sentido contrario al campo magnético interno. Este campo magnético, asociado con el campo eléctrico sobre la superficie externa de las placas resistivas da lugar a un vector de Poynting entrante en la superficie externa de las placas resistivas. Si la dimensión a lo largo del eje $x$ se hace cada vez más pequeña, el campo magnético en el exterior crece y su módulo se hace comparable al del campo magnético interno. En un hilo resistivo cilíndrico, por lo tanto, el flujo entrante es aproximadamente el mismo sobre todos los puntos de la superficie.

\section{Análisis cualitativo de la carga super- ficial y el campo eléctrico en un cir- cuito más realista}

En la mayoría de las situaciones prácticas, o por lo menos en los circuitos que se muestran en los textos, los conductores y resistencias son hilos y no placas. ¿Qué sucede si las placas resistivas de la Fig. 2 se reemplazan por un par de hilos cilíndricos como se muestra 
en la Fig. 5? Assis y Mania [15] estudian los campos eléctricos y magnéticos que rodean a los hilos, un problema que está sugerido en el clásico texto de Stratton [16]. La solución analítica de este problema, debido a su nivel de dificultad, no puede abordarse en un curso básico de física, aún de nivel universitario. Sin embargo, de acuerdo con los resultados de la sección anterior, se puede concluir que la distribución de cargas superficiales sobre los conductores resistivos es como la que se muestra en la Fig. 5 . El gradiente de carga superficial sobre los hilos resistivos crea un campo eléctrico, que se distribuye uniformemente sobre sus secciones en la dirección que fluye la corriente. Un electrón libre que está en la parte superior del conductor cilíndrico de la izquierda será atraído hacia la parte inferior donde las cargas superficiales positivas tienen una mayor concentración, mientras que un electrón que está en la parte inferior del cilindro de la derecha será repelido hacia la zona de más baja concentración de cargas negativas de la parte superior.

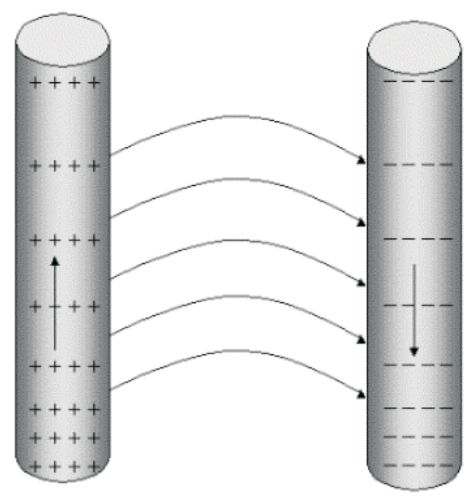

Figura 5 - Distribución de las cargas superficiales a lo largo de los hilos que forman el circuito y las líneas del campo eléctrico en el plano definido por los dos hilos.

El campo eléctrico en el exterior de los hilos y en particular en el plano definido por ambos, tiene aún el aspecto de la Fig. 3.

Reconociendo que los campos eléctricos en el interior de los conductores son creados por las cargas superficiales se consigue también explicar, al menos, cualitativamente, cómo el campo eléctrico cambia su dirección cuando el circuito se curva como se muestra en la Fig. 6. Imaginemos que un electrón que se mueve a lo largo del hilo se acerca hacia el codo. Este electrón podrá doblar el codo si es empujado o atraído por cargas que están sobre la superficie del hilo. Si esto no ocurre, el electrón llega hasta la superficie del codo modificando la carga superficial en esa zona. De este modo el primer electrón que no pudo dar la vuelta ayuda a empujar al que viene detrás para que éste sí lo haga. Este proceso continúa hasta que la distribución de carga superficial que se construye es el adecuado para encauzar a todos los electrones de conducción a lo largo de los hilos, esto es, en líneas paralelas a la superficie del conductor.

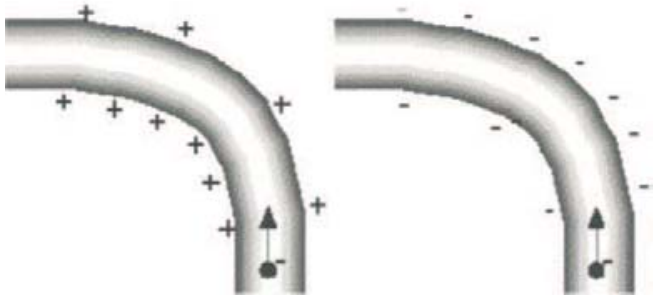

Figura 6 - Las cargas superficiales en los codos del circuito se distribuyen como se muestra en la figura para producir en el interior del hilo el campo adecudo para que los electrones de conducción contorneen el hilo.

\section{Análisis cualitativo del flujo de en- ergía en un circuito real}

El cálculo de los campos eléctricos y magnéticos que rodean a los circuitos reales es una tarea muy difícil y es generalmente imposible encontrar soluciones analíticas. Sin embargo, como vimos en la sección 4, las soluciones exactas que se obtienen con geometrías simples e ideales ayudan a inferir cómo son estos campos en geometrías más complicadas y permiten esbozar un dibujo cualitativo de sus líneas de fuerza.

La Fig. 7 muestra la representación usual de un circuito formado por una batería y una resistencia conectadas por dos hilos perfectamente conductores. Los hilos se cargan, el superior con cargas positivas y el inferior con cargas negativas. La distribución de cargas superficiales, en este caso, tiene un gradiente nulo y, por lo tanto, el campo eléctrico en el interior de los hilos es nulo. Las líneas del campo eléctrico salen normalmente del hilo cargado positivamente y terminan normales en la superficie del hilo cargado negativamente como se muestra esquemáticamente en la Fig. 7. El campo eléctrico, sin embargo, tiene una componente tangencial a lo largo de la resistencia.
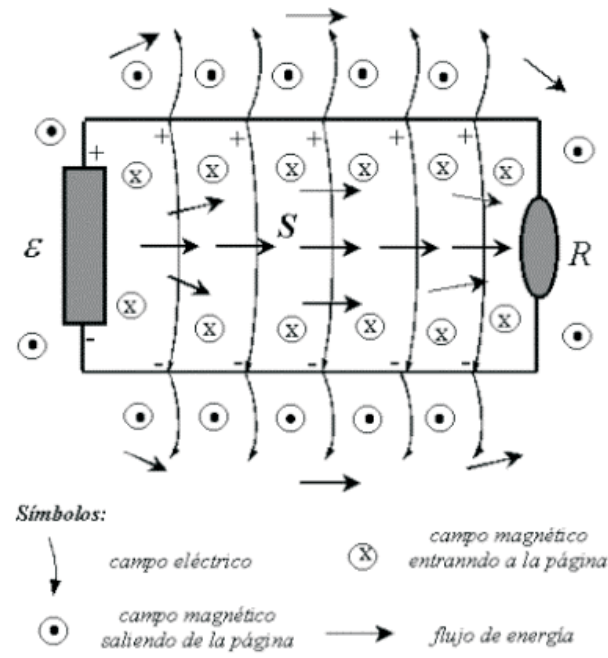

Figura 7 - Esquema que muestra las direciones de los campos eléctricos y magnéticos y el flujo de energía en el plano del circuito. 
La corriente que circula en el circuito crea un campo magnético, cuyas líneas de fuerza son perpendiculares al plano del circuito, entrantes en su zona interna y salientes en su zona externa.

Con esta información se puede estimar el módulo, dirección y sentido del vector flujo de energía en diferentes puntos del plano. Se encuentra que las trayectorias del flujo de energía salen de la batería y se dirigen a la resistencia en un tránsito de una sola vía, en la dirección correcta.

\section{Discusión}

El análisis de circuitos en términos de cargas superficiales y campos da respuestas a algunas de las preguntas que no tienen una explicación apropiada dentro del contexto de la teoría de circuitos tradicional: ¿Quién crea el campo eléctrico que mueve las cargas en el interior de los conductores donde circula una corriente?, ¿Porqué el campo eléctrico cambia su dirección de un tramo a otro del circuito? ¿Cómo fluye la energía desde la batería hasta los elementos resistivos donde se disipa? No se pueden responder estas preguntas en el marco de la teoría de circuitos tradicional que se basa en el concepto de diferencia de potencial. El gradiente de la densidad de carga superficial, en la superficie de los elementos resistivos, crea el campo eléctrico que produce la corriente que circula en su interior. Es este campo eléctrico el origen de la diferencia de potencial a lo largo del elemento resistivo. En consecuencia, este análisis, considera fundamental el concepto de campo eléctrico y a la diferencia de potencial un concepto derivado. Las cargas superficiales, que son requeridas para mantener la corriente, producen también, en el espacio exterior de los conductores, el campo eléctrico que se necesita para la transferencia de energía desde la batería hasta la resistencia.

A primera vista, los argumentos desarrollados en este trabajo pueden dar la impresión de ser difíciles. Sin embargo, las principales ideas no son más complicadas que el concepto de campo que se introduce en casi todos los cursos de física universitaria. En cualquier caso el campo es un concepto fundamental, aún más básico que el concepto de energía, de modo que se hace difícil pen- sar como podemos estudiar física sin tenerlo en cuenta. Los argumentos cualitativos que presentamos son similares a los que se utilizan cuando se presenta la luz como una onda electromagnética, debido a que los conceptos esenciales que se necesitan son los mismos en ambos casos. El desafío se puede plantear de esta manera: si este material parece difícil ¿de qué otra manera se lo puede enseñar sin introducir errores conceptuales?

\section{Referências}

[1] J.D. Jackson, Am. J. Phys. 64, 855 (1996)

[2] W.T. Scott, The Physics of Electricity and Magnetism (J. Wiley \& Sons, N.Y., 1966).

[3] A. Sommerfeld, Electrodynamics (Academic Press, New York, 1952).

[4] J.R. Reitz and F.J. Milford, Foundations of Electromagnetic Theory (Addisson-Wesley, Inc. Massachusets, 1966).

[5] R.W. Chabay and B.A. Sherwood, Electric and Magnetic Interactions (J. Wiley \& Sons, New York, 1999).

[6] H. Haertel, en Aspects of Understanding Electricity: Proceedings of an International Conference, editado por R. Duit, W. Jung and C. von Rhöneck (IPN/Schmidt \& Klaunig, Kiel, Alemania, 1985).

[7] N.W. Prayer, Am. J. Phys. 68, 1002 (2000).

[8] B.R. Russell, Am. J. Phys. 36, 527, (1968).

[9] G.F. Leal Ferreira e M.T. de Figueiredo, Rev. Bras. Ens. Fís. 25, 374, (2003).

[10] O.D Jefimenko, Electricity and Magnetism (Electrect Scientific Company, Star City, 1989) $2^{\text {nd }}$ ed.

[11] A.K.T. Assis, J.A. Hernandez and J.E. Lamesa, Foundations of Physics 31, 1501 (2001).

[12] A.K.T. Assis, W.A. Rodrigues Jr. and A.J. Mania, Foundations of Physics 29, 729 (1999).

[13] M. Heald, Am.J.Phys. 52, 522 (1984).

[14] J.A. Hernandez and A.K.T. Assis, Phys. Rev. E 68, 046611 (2003).

[15] A.K.T. Assis e A.J. Mania, Rev. Bras. Ens. Fis. 21, 469 (1999)

[16] J.A. Stratton, Electromagnetic Theory (McGraw-Hill, New York, 1941). 